\title{
Implantação do Núcleo de Apoio à Saúde da Família: percepção do usuário
}

Implementation of the Family Health Support Units: the user's perception

Fernando Leonardo Diniz Souza', Eduardo Paul Chacur², Maura Regina Guimarães Rabelo³, Luciana de Araújo Mendes Silva ${ }^{4}$, Wilza Vieira Villela 5 .

' Doutorando em Promoção de Saúde pela Universidade de Franca (UNIFRAN) - Franca (SP), Brasil. Docente do Departamento de Saúde da Faculdade Patos de Minas (FPM) Patos de Minas (MG), Brasil.

fernandoldiniz@yahoo.com.br

2 Doutorando em Promoção de Saúde pela Universidade de Franca (UNIFRAN) - Franca (SP), Brasil. Docente do Curso de Medicina do Centro Universitário de Patos de Minas (UNIPAM) - Patos de Minas (MG), Brasil. eduardopc@unipam.edu.br

${ }^{3}$ Especialista em Medicina de Família e Comunidade pela Sociedade Brasileira de Medicina da Família e Comunidade (SBMFC) - Rio de Janeiro (RJ), Brasil. Coordenadora e Docente do Curso de Medicina do Centro Universitário de Patos de Minas (UNIPAM) Patos de Minas (MG), Brasil. maura@unipam.edu.br

${ }^{4}$ Mestre em Promoção de Saúde pela Universidade de Franca (UNIFRAN) - Franca (SP), Brasil. Docente do Departamento de Saúde da Faculdade Patos de Minas (FPM) Patos de Minas (MG), Brasil. laraujo3@yahoo.com.br

${ }^{5}$ Doutora em Medicina Preventiva pela Universidade de São Paulo (USP) - São Paulo (SP), Brasil. Docente do Programa de Pós-Graduação em Promoção da Saúde pela Universidade de Franca (UNIFRAN) - Franca (SP), Brasil. Programa de Pós-Graduação em Saúde Coletiva da Universidade Federal de São Paulo (UNIFESP) - São Paulo (SP), Brasil. Livre-docente em Saúde Coletiva da Universidade Federal de São Paulo (UNIFESP) - São Paulo (SP), Brasil.

wilsa.vieira@terra.com.br
RESUMO $O$ artigo discute como os usuários de um Núcleo de Apoio à Saúde da Família (NASF) compreendem esta forma de atendimento. Foi realizada pesquisa qualitativa, de caráter exploratório, identificando a visão do usuário a respeito da atuação do NASF. Foram entrevistadas doze mulheres, selecionadas a partir da sua inserção em alguma das atividades oferecidas. As usuárias entendem que as atividades realizadas pelo NASF melhoram sua saúde, mas não identificam o programa como uma atividade oferecida pelo Sistema Único de Saúde (SUS). Pode-se concluir que a implantação de um serviço multiprofissional voltado para a atenção primária à saúde deve ser fortalecida de modo a consolidar uma proposta de atenção integral à saúde contida no SUS.

PALAVRAS CHAVE: Saúde da Família; Atenção Primária à Saúde; Promoção da Saúde; Sistema Único de Saúde.

\begin{abstract}
This paper discusses how the users of Family Health Support Units (NASF) perceive this form of assistance. An exploratory qualitative survey was conducted to identify the users' views on the actions of the NASF System. Interviews were held with twelve women, who were selected based on their participation in activities offered by the program. The female users acknowledged that the activities promoted by the NASF improve their health but did not identify the program as an activity provided by the SUS. It can be concluded that the implementation of a multidisciplinary service focused on primary health care should be strengthened in order to consolidate the proposal for comprehensive public health care.
\end{abstract}

KEYWORDS: Family Health; Primary Health Care; Health Promotion; Unified Health System. 


\section{Introdução}

Após a adoçâo da Estratégia Saúde da Família (ESF) como modelo de atenção para a reorganização e o fortalecimento da atençâo básica em saúde no Brasil, as ações do Sistema Único de Saúde (SUS), neste nível de atenção, têm buscado o fortalecimento das intervençóes multiprofissionais voltadas para a promoção da saúde. Nesta perspectiva foram criados os Núcleos de Apoio à Saúde da Família (NASF), com o objetivo de apoiar e ampliar a atenção e a gestão da saúde na Atenção Básica e Saúde da Família. O propósito dos NASF é oferecer açóes de promoção e atenção à saúde tecnicamente orientadas para contribuir com as açóes da ESF (BRASIL, 2008). Cada NASF tem sob sua responsabilidade o atendimento a usuários de um determinado número de equipes de Saúde da Família, ou seja, os profissionais do NASF atuam nas suas especialidades para complementar a ação das equipes de Saúde da Família às quais estão vinculadas.

O início do processo da implantação de atividades não médicas e multiprofissionais no âmbito da atenção primária em saúde deu-se em 2005, com a inserção de profissionais de diferentes áreas nas equipes de Saúde da Família. A finalidade foi construir coletivamente práticas de saúde frente aos problemas identificados em uma dada comunidade. Foram propostas quatro modalidades de ação: alimentação/nutrição; atividade física; saúde mental e reabilitação (BRASIL, 2005). Posteriormente, esta proposta foi reformulada para dar origem à criação dos NASF em sua versão atual. Isso flexibilizou a composição da equipe multiprofissional (AVEIRO et al, 2011). Levando em conta critérios relativos à demanda local, os NASF não são porta de entrada ao sistema, mas apoio multidisciplinar ao mapeamento de agravo e prevenção realizados pelo Programa Saúde da Família (PSF) (BRASIL, 2008).

O NASF, em contraste com os modelos convencionais de prestação de cuidados, busca operar numa lógica de corresponsabilização e gestão integrada do cuidado, por meio de atendimentos e projetos terapêuticos que envolvam os usuários e considerem a singularidade dos sujeitos assistidos (NASCIMENTO; OLIVEIRA, 2010).

\section{A implantação do NASF em um município de médio porte em Minas Gerais}

O município em que foi realizada a pesquisa cujos resultados são tema deste artigo conta com cerca de 136 mil habitantes, apresentando um Índice de Desenvolvimento Humano (IDH) de 0,813 (IBGE, 2010). Três equipes de NASF foram implantadas em agosto de 2008, cada uma contando com assistente social, educador físico, fisioterapeuta, nutricionista e psicólogo, com a responsabilidade de atender a todo o município.

Após a contratação, os profissionais das três equipes reuniram-se por nove semanas consecutivas para planejar suas atividades de modo integrado, sendo estas apresentadas aos coordenadores de cada unidade da ESF. A partir de entáo, os NASF passaram a desenvolver atividades multidisciplinares focadas na atenção primária, envolvendo grupos de usuários em todo o município, incluindo a zona rural. De acordo com a categoria profissional, estas atividades compreenderam: caminhada orientada (educadores físicos e fisioterapeutas), exercícios preventivos para coluna vertebral (fisioterapeutas), controle de fornecimento de oxigênio domiciliar (fisioterapeutas), visitas domiciliares para orientação dos cuidadores (fisioterapeutas, nutricionistas, assistentes sociais), orientação alimentar (nutricionistas), fortalecimento muscular (educadores físicos e fisioterapeutas), orientaçóes familiares (psicólogos e assistentes sociais) e terapia comunitária (multiprofissional), além de cursos de gestantes e cuidadores (multiprofissional).

Considerando que a oferta deste tipo de atividades e a incorporação destes profissionais é uma prática inovadora dentro das açôes de atenção primária à saúde tradicionalmente realizadas no País, após um ano de trabalho, buscou-se conhecer como os usuários do programa percebiam esta proposta.

Sendo as açóes multiprofissionais atividades novas inseridas na comunidade onde a ESF atua em uma vertente predominantemente assistencial, com marcaçóes e realizaçôes de consultas e encaminhamentos para exames complementares, interessa saber qual a percepção do usuário em relação a uma prática de saúde distinta daquela habitual. Supóe-se que conhecer a percepção dos usuários destas novas práticas em saúde pode ser útil 
para nortear melhor as propostas do NASF, bem como subsidiar açóes que contribuam para promover uma visão positiva de saúde, ou seja, o modo como os usuários do NASF estão vivenciando uma oferta de açóes em saúde por profissionais não médicos, que têm por finalidade a promoção, a preservação e a recuperação da saúde na perspectiva da atenção primária à saúde.

\section{Metodologia}

Considerando-se os distintos significados atribuídos à ideia de saúde e do processo de adoecer, e ainda as diferentes concepçôes a respeito dos fatores que interferem na dinâmica saúde/doença, foi realizado um estudo qualitativo, de caráter exploratório, visando a identificar a percepção dos usuários do NASF a respeito desta proposta (MINAYO, 2007).

Assumiu-se que a percepção dos usuários manifesta a forma como estes entendem as intervençóes em saúde e sustenta-se na capacidade que o indivíduo apresenta para a construção e a execução das ações em promoção de saúde. A percepção das pessoas sobre uma proposta de ação pode ser uma relevante ferramenta para a análise da sua adequação às necessidades dos sujeitos.

O estudo foi realizado na área de uma das equipes de PSF do município com participantes de atividades desenvolvidas pelo NASF. A seleção desta equipe foi realizada através de sorteio simples, em que foram incluídas as dez equipes de PSF que apresentam maior número de usuários inseridos nas atividades multidisciplinares do NASF. A equipe sorteada atua em uma Unidade Básica de Saúde (UBS) que combina, no mesmo espaço físico, o atendimento tradicional das unidades de saúde e atividades do PSF. O bairro encontra-se com certa dificuldade estrutural em vias públicas e espaços de lazer, a população predominante apresenta-se em nível socioeconômico baixo.

Para a seleção dos sujeitos de pesquisa foi afixado no quadro de avisos da unidade de saúde um cartaz convidando os usuários a participarem de um estudo sobre a atuação do NASF no município. Por este processo, 21 pessoas apresentaram-se como voluntárias.
Estes sujeitos foram convidados a realizar uma entrevista sempre agendada para depois da execução da atividade na qual eles estavam inseridos. Destas entrevistas agendadas, 18 foram realizadas. Foi utilizada, como instrumento de coleta de dados, entrevista semiestruturada a partir de roteiro voltado para buscar captar a percepção do sujeito sobre a atuação do referido programa e o contato com profissionais não médicos.

O roteiro foi elaborado a partir da observação de grupos realizados pelos profissionais do NASF e de investigações sobre dúvidas e comentários dos usuários participantes. As questões foram agrupadas em dois eixos principais, da seguinte forma: inicialmente, buscouse contextualizar os aspectos da saúde no passado com o modelo atual; depois, foram questionadas as características da participação nas atividades do NASF, o contato com profissionais não médicos e o impacto disso na vida dos entrevistados.

As entrevistas foram realizadas pelos pesquisadores, individualmente, em local reservado definido pelo entrevistado, no período de maio a julho de 2010. Todas as entrevistas foram gravadas em áudio e transcritas na íntegra.

Após a transcrição, foi feita uma leitura exaustiva do material, buscando-se estabelecer as categorias emergentes das falas em relação aos assuntos tratados na entrevista. A análise do material foi realizada buscando-se identificar recorrências e dessemelhanças em relação a cada tópico da entrevista.

O estudo foi aprovado pelo Comitê de Ética e Pesquisa (CEPE) da Universidade de Franca (UNIFRAN), sob o parecer $n^{\circ}$ 0022/2010. Todos os sujeitos da pesquisa assinaram um termo de consentimento informado contendo os objetivos da pesquisa e esclarecendo seus direitos.

\section{Resultados e Discussão}

Do total de 18 entrevistas realizadas, 6 foram posteriormente recusadas para análise em função de problemas com a gravação ou dificuldades na condução da entrevista. Desta forma, os resultados apresentados se referem à análise de 12 entrevistas. Todas as participantes 
eram do sexo feminino, o que é semelhante ao perfil da maioria dos atendimentos realizados pela equipe estudada. A faixa etária das 12 usuárias variou entre 46 e 73 anos. A maioria das entrevistadas náo exerce atividade remunerada fora do lar atualmente, sendo que quatro - o equivalente a 3,3\% - já desempenharam atividades como trabalhadora rural ou doméstica; cinco - o equivalente a $41,6 \%$ - têm como fonte de renda a aposentadoria; sete - o equivalente a $58,3 \%$ - são casadas; e três - o equivalente a $25 \%$ - são viúvas, solteiras ou separadas. A escolaridade das entrevistadas variou de 0 a 15 anos de estudo. Estas características correspondem a estudos realizados no Nordeste do Brasil, na ocasiáo em que foi investigado o perfil epidemiológico de mulheres atendidas em grupos de apoio para incapacidade funcional (MELO; SAINTRAIN, 2009).

\section{Percepção dos usuários sobre a atuação do NASF na comunidade}

Perguntadas sobre o que entendiam das atividades do NASF, o primeiro fato que chamou atenção foi o total desconhecimento por parte do conjunto das entrevistadas, à exceção de uma, da sigla NASF ou do seu significado. Esta falta de nomeação não impede a participação nas atividades, mas pode comprometer a posterior articulação entre as atividades realizadas e uma proposta de saúde. Os depoimentos seguintes mostram a desvinculação, para as usuárias, entre as ações do NASF e as ofertas do sistema de saúde.

[...] do NASF, não! Eu só sei que 'táo acontecendo essas atividades aqui no barracão, caminhada lá em baixo, palestra com a nutricionista. Eu já fui até nas rodas de terapia (USUÁRIA 4).

Em um estudo sobre percepção da qualidade de vida, realizado com usuários do PSF em 2009, as autoras discutem que a maioria do trabalho do PSF e do SUS ainda é direcionado para açóes de caráter curativo que talvez náo resultem na melhora da qualidade de vida da população. Salientam ainda que se faz necessária a realização de atividades voltadas para a promoção de saúde em todo seu amplo espectro (MAIA; SANTOS, 2011). As ações em promoção de saúde devem ser a base das atividades do SUS. A falta de mobilização neste aspecto gera um modelo de saúde travado nas açóes curativas, aumentando demandas e paralisando o processo. Este tipo de mobilização deve partir dos profissionais envolvidos no desenvolvimento da sustentabilidade do processo de saúde, dando poder à população, para que atividades saudáveis sejam tidas como obrigação de todos os membros da comunidade. Assim, falta de identificação da sigla NASF também indica a falta de divulgação de tais atividades dentro do escopo do PSF.

Após esclarecimento sobre o que é o NASF, quais atividades são desenvolvidas, quais profissionais estão atuando e seus vínculos com as atividades do PSF da região, várias participantes demonstraram surpresa e logo retomaram a questão, apresentando uma avaliação positiva.

[...] Então, eu sei o que é, só que não juntava o nome com o que o pessoal faz aqui no Alvorada. Até foi uma amiga minha que me chamou pra vim fazer ginástica e caminhada. Eu vi mesmo que, d'um tempo pra cá, tem muita gente fazendo caminhada lá em baixo, mas eu não sabia que tinha a ver com o postinho, não (USUÁRIA 5).

[...] Ah, depois desse NASF, então, hoje minha vida é outra: eu procuro fazer a caminhada lá na avenida com o professor, vou nas palestras da nutricionista, venho aqui na ginástica. Eu procuro, nessas atividades, fazer minhas coisas, meu serviço de casa, dormir bem, me alimentar bem. E algumas dessas coisas, comecei a fazer depois que esse negócio de NASF veio aqui pro Alvorada (USUÁRIA 1).

Algumas fragilidades apontadas pelas usuárias, como a falta de divulgação do programa, figuram como um fato importante para o direcionamento de novas açóes, o aumento da cobertura e o treinamento de atores envolvidos no processo de saúde. 
O encaminhamento dos usuários para a maioria das atividades não passa pela unidade de saúde local. É mais frequente, segundo as usuárias, que os convites sejam feitos por vizinhas ou amigas. Em estudo realizado na Austrália, a variável "receber convite de amigos" para a prática de atividade física foi significativa. $\mathrm{Na}$ amostra estudada, reflete a náo adesão dos indivíduos aos programas oferecidos pelos sistemas de saúde (BALL et al, 2001).

Faz-se importante lembrar que o NASF não é porta de entrada ao SUS, o fluxo de encaminhamento deve ser oriundo da unidade de saúde local. Apesar deste fluxo não ser ideal, porque fragmenta o processo de intervenção, as usuárias percebem que as atividades oferecidas pelo NASF colaboram para o bem-estar de parte da comunidade, entretanto, necessário se faz destacar a falta de divulgação pelos componentes das equipes de saúde.

\section{[...] Então! Eu acho que tudo isso que está acontecendo faz bem pro povo demais, tira o povo de casa. Só acho que num é todo mundo que sabe disso tudo que 'tá acontecendo não... (USUÁRIA 8).}

Entre estes atores importantes envolvidos no processo de divulgação do programa, os Agentes Comunitários de Saúde (ACS) destacam-se por estar em contato direto com os usuários das comunidades. A percepção dos ACS, segundo Hildebrand e Shimizu (2008), em estudo realizado sobre o Programa Saúde da Família, demonstra que o trabalho em equipe multidisciplinar, com atuação interdisciplinar, está pautado nos seguintes critérios: articulação das açóes, reconhecimento das diferenças técnicas entre os trabalhos especializados, questionamento das desigualdades estabelecidas entre os diversos trabalhadores, função de cada um dentro do processo de trabalho e, principalmente, comunicação entre os agentes de trabalho. Caso estes critérios náo estejam bem claros para toda a equipe, as intervençóes podem não ter a efetividade e a cobertura desejada.

Este elemento de negação inicial é um fato importante para a contextualização da percepção das usuárias sobre os serviços prestados pelo NASF na região estudada, pois existe entendimento, por parte das usuárias, a este respeito. Porém, em suas concepçōes, os serviços não apresentam ligação com as atividades do PSF, sendo que, na verdade, o NASF dá apoio interdisciplinar às equipes de saúde da família na tentativa de gerar sustentabilidade na esfera da atenção primária à saúde. A percepção negativa da saúde é um fato intrínseco à população com idade avançada. Em pesquisa, Silva et al (2012) revelam que as limitações das atividades de vida diária, em alguns casos, associadas a problemas socioeconômicos e demográficos, fazem com que os indivíduos tenham opinião pessimista em relação ao seu estado de saúde. Em um estudo sobre a percepção dos usuários em atenção básica à saúde, observou-se, nos resultados, que, quanto à modalidade de serviços, os usuários não percebem diferença entre centros de saúde tradicionais e equipes de saúde da família (VAN STRALEN et al, 2008).

Para Villela et al (2009), a ideia de integralidade do acesso aos serviços de saúde, com ênfase na atenção primária, exige o reconhecimento do usuário que busca o serviço de saúde e que é um sujeito inserido numa complexa trama psicossocial, na qual sua queixa inscreve-se e adquire um sentido particular e único.

O NASF é um projeto ambicioso e pode parecer utópico quando avaliado que o perfil formativo da maior parte dos profissionais de saúde ainda está muito distante desse horizonte de atenção primária e que muitos obstáculos serão encontrados no percurso (MOLINI-AVEJONAS; MENDES; AMATO, 2010).

A evolução dos modelos de atenção propóe mudanças nas características de modelos assistenciais já existentes e diferenciam-se quanto à organização do serviço e do trabalho em saúde, revelando a necessidade de chamar os profissionais de saúde à reflexão sobre o seu trabalho e o modelo assistencial que lhes é subjacente. Na medida em que este trabalho tem se constituído em consonância com os princípios do SUS, a percepção dos usuários do NASF norteia uma nova abordagem no espectro da atenção primária à saúde (BEZERRA et al, 2011).

Programas de atividade física orientados e diferenciados em caminhadas, alongamento e fortalecimento com periodicidade semanal, inseridos na atenção 
primária à saúde, melhoram a dinamicidade de mulheres acima de 60 anos. Verifica-se, também, diminuição da incidência de doenças crônicas, como diabetes e hipertensão, além de melhorar os níveis de atividade motora em pessoas idosas (MOLINA-SOTOMAYOR, GONZÁLEZ-JURADO, LÉON-PRADOS, 2010; ALENCAR et al, 2011). A atividade física, quando executada continuamente por, pelo menos, 30 minutos diários, revela uma melhora significativa na qualidade de vidas das pessoas, provocando efeitos favoráveis nos níveis psicológico, ambiental e social (GUIMARÁES; BAPTISTA, 2011).

Estas situações, quando analisadas em longo prazo, podem minimizar o impacto de demanda contínua na rede de saúde e ainda estimular novos investimentos na base da atenção primária.

Para que haja efetividade de práticas que envolvem atividade física em nível de atenção primária dentro do escopo do PSF, os espaços físicos públicos e privados utilizados devem ter as condiçóes mínimas necessárias para uma boa condução do trabalho, e agregação social dos indivíduos envolvidos: profissionais e usuários (SALVADOR et al, 2009; HALLAL et al, 2010).

\section{O contato com profissionais não médicos}

$\mathrm{Na}$ tentativa de buscar a melhor contextualização para entender a percepção global das voluntárias, o próximo passo da entrevista foi buscar a opiniāo das voluntárias sobre o contato com profissionais não médicos, a partir das atividades oferecidas pelo núcleo.

$\mathrm{Na}$ fala das voluntárias percebeu-se que as opinióes foram positivas em relação às atividades não médicas oferecidas, partindo do princípio que tais atividades nunca existiram naquela região. Talvez isso chame a atenção das usuárias, segundo a fala da voluntária 10 sobre os serviços oferecidos.

[...] Eu acho muito bom pro povo sair de casa. Tem muita gente que só fica dentro de casa. A prefeita tinha que ter feito isso antes, sabe? Pode ver: hoje tinha tanta gente aqui fazendo ginástica... E, se vocêpensar, esse povo num tem muita condição de pagar pra fazer essa ginástica pra coluna, não, né?! (USUÁRIA 10).

Sobre este elemento, houve saturação nas falas das usuárias, no sentido positivo, visto que todas participam espontaneamente de atividades oferecidas pelo NASF, porém, com ressalvas, segundo a fala da voluntária 10, pois a difusão de informações sobre prestação de serviços não apresenta cobertura adequada.

Em um estudo sobre a satisfação do usuário do PSF, os resultados demonstram que existe certo grau de satisfação por parte dos mesmos, sendo fatores significativos desta satisfação os seguintes itens: maior acesso aos cuidados médicos, melhoria do nível de informação sobre o processo saúde-doença (situação de risco e proteção, cuidados básicos), a existência da visita domiciliar como elemento-chave da prevenção e do acompanhamento, oferecimento de grupos direcionados e acompanhamento por equipes multidisciplinares (TRAD et al, 2002).

$\mathrm{Na}$ busca pelo entendimento de questōes que revelam a percepção das usuárias sobre os serviços de saúde prestados na esfera da atenção primária, os diálogos desenvolvidos durante as entrevistas revelam, dentro do contexto da palavra, que há percepção das usuárias sobre a importância de atividades que norteiam cuidados e ensinamentos para que haja melhoria na vida das pessoas, mesmo que os programas náo sejam muito bem entendidos pela população.

\section{Considerações Finais}

Conclui-se que a implantação de um serviço multidisciplinar voltado à atenção primária à saúde tem impacto na percepção das pessoas por ser uma estratégia pioneira no âmbito do Sistema Único de Saúde. O fortalecimento destas práticas pode incrementar o modelo de atenção integral ao indivíduo, sendo, desta forma, necessária uma intervenção educativa maior.

Nos diálogos, observou-se avanço no que tange à tecnologia, ao aumento do número de profissionais médicos e não médicos, à gratuidade dos serviços 
prestados; todavia, algumas ressalvas foram significativas nos discursos. As voluntárias, em sua maioria, percebem que o aumento da população em geral e a migração do campo para as cidades dificultam o avanço deste processo, levando ao surgimento de alguns problemas, tais como filas e demora para atendimentos e exames específicos. As usuárias, de acordo com os resultados, entendem que as atividades propostas pelo NASF, de alguma maneira, fazem parte do cotidiano de suas vidas com uma boa perspectiva, elevando a amplitude de atividades de vida diária executadas.
Os diálogos, no entanto, demonstram que as ações já desenvolvidas interferem nas concepções do cuidado preventivo com a saúde, provocando mudanças significativas no cotidiano individual e familiar com relação à atenção primária à saúde. Porém, ficou claro que tais intervençóes necessitam de maior divulgação entre a comunidade, visto que a cobertura do atendimento multiprofissional ainda é pequena.

Os fatos descritos neste estudo levantam a necessidade de novas exploraçóes para identificar a adesão e o fluxo de encaminhamento de novos usuários para o NASF, buscando contribuir com a melhora da cobertura do SUS.

\section{Referências}

ALENCAR, N. A. et al. Nível de atividade física em idosas. Revista Brasileira de Promoção da Saúde, Fortaleza, v. 24, n. 3, p. 251-257, jul./set. 2011. Disponível em: <http://www.unifor .br/images/ pdfs/rbps/artigo9_2011.3.pdf>. Acesso em: 10 jan. 2012.

AVEIRO, M. C. et al. Perspectivas da participação do fisioterapeuta no Programa Saúde da Família na atenção à saúde do idoso. Ciência e Saúde Coletiva, Rio de Janeiro, v. 16, supl., p. 1467-1478, 2011. Disponível em: <http://www.scielo.br/pdf/csc/v16s1/ a82v16s1.pdf>. Acesso em: 19 mar. 2012.

BALL, K. et al. Perceived environmental aesthetics and convenience and company are associated with walking for exercise among Australian adults. Preventive Medicine, New York, v. 33, p. 434440, nov. 2001. Disponível em: <http://www.ncbi.nlm.nih.gov / pubmed/11676585>. Acesso em: 8 dez. 2011

BEZERRA, I. M. P. et al. Velhas e novas formas de intervenção em saúde: os modelos assistenciais em análise. Revista Saúde em Debate, Rio de Janeiro, v. 35, n. 4, p. 197-206, abr./jun. 2011. Disponível em: <http://www.sumarios.org/sites/default/files/ pdfs/sdv35n89_02.pdf>. Acesso em: 9 dez. 2011.

BRASIL. Ministério da Saúde. Portaria $n^{\circ}$ 154, de 24 de janeiro de 2008. Cria os Núcleos de Apoio à Saúde da Família. Brasília: Ministério da Saúde, 2008.

Portaria n 1065, de 4 de julho de 2005. Cria os Núcleos de Atenção Integral na Saúde da Família. Brasília: Ministério da Saúde, 2005.
GUIMARÃES, A. C. A.; BAPTISTA, F. Atividade física habitual e qualidade de vida de mulheres na meia-idade. Revista Brasileira de Medicina do Esporte, São Paulo, v. 17, n. 5, p. 305-309, set./out. 2011. Disponível em: <http://www.scielo.br/pdf/rbme/v17n5/ a02v17n5.pdf>. Acesso em: 12 jan. 2012.

HALLAL, P. C. et al. Avaliação do Programa de Promoção da Atividade Física Academia da Cidade de Recife, Pernambuco, Brasil: Percepções de Usuários e não usuários. Cadernos de Saúde Pública, Rio de Janeiro, v. 26, n. 1, p. 70-78, jan. 2010. Disponível em: <http://www.scielo.br/pdf/csp/v26n1/08.pdf>. Acesso em: 10 fev. 2012.

HILDEBRAND, S. M.; SHIMIZU, H. E. Percepção do agente comunitário sobre o Programa Família Saudável. Revista Brasileira de Enfermagem, Brasília, v. 61, n. 3, maio/jun. 2008, p. 319-324.

INSTITUTO BRASILEIRO DE GEOGRAFIA E ESTATÍSTICA. Banco de dados. Disponível em: <http://www.ibge.gov.br/cidadesat/ topwindow.htm>. Acesso em: 30 ago. 2010.

MAIA, M. A. C.; SANTOS, J. S. Qualidade de vida no programa saúde da família: a visão de agentes comunitários de saúde e usuários. Revista Brasileira de Promoção da Saúde, Fortaleza, v. 24, n. 3, p. 228237, jul./set. 2011. Disponível em: <http://www.unifor.br/images/ pdfs/rbps/artigo6_2011.3.pdf>. Acesso em: 12 fev. 2012.

MELO, N. L. P.; SAINTRAIN, M. V. L. Perfil epidemiológico de mulheres idosas atendidas no Grupo de Apoio à Prevenção da Incapacidade Funcional. Revista Brasileira de Promoção da Saúde, 
Fortaleza, v. 22, n. 4, p. 251-258, out./dez. 2009. Disponível em: <http://www.unifor.br/images/pdfs/rbps/artigo7_2009.4.pdf>. Acesso em: 15 jan. 2012.

MINAYO, M. C. S. O desafio do conhecimento: pesquisa qualitativa em saúde. São Paulo: Hucitec, 2007.

MOLINA-SOTOMAYOR, E.; GONZÁLEZ-JURADO, J. A.; LÉONPRADOS, J. A. Efectos de los programas de entrenamiento sobre aptitud física metabólica em adultos mayores. Hacia la Promoción de la Salud, Manizales, v. 15, n. 2, p. 45-63, jul./dic. 2010. Disponível em: <http:/promocionsalud.ucaldas.edu.co/downloads/ Revista15(2)_4.pdf>. Acesso em: 8 abr. 2012.

MOLINI-AVEJONAS, D. R.; MENDES, V. L. F; AMATO, C. A. H. Fonoaudiologia e Núcleos de Apoio à Saúde da Família: conceitos e referências. Revista da Sociedade Brasileira de Fonoaudiologia, São Paulo, v. 15, n. 3, p. 465-474, jul./set. 2010. Disponível em: <http:// www.scielo.br/pdf/rsbf/v15n3/24.pdf>. Acesso em: 10 dez. 2011.

NASCIMENTO, D. D. G.; OLIVEIRA, M. A. C. Reflexões sobre as competências profissionais para o processo de trabalho nos Núcleos de Apoio à Saúde da Família. O mundo da Saúde (CUSC), São Paulo, v. 34, n. 1, p. 92-96,jan./mar. 2010. Disponível em: <http:// www.saocamilosp.br/novo/publicacoes/publicacoesDowload. php?|D=74\&rev=s\&ano=2010>. Acesso em: $10 \mathrm{dez} .2011$.

SALVADOR, E. P. et al. Percepção do ambiente e prática de atividade física no laser entre idosos. Revista de Saúde Pública, São Paulo, v.
43, n. 6, p. 972-980, dez. 2009. Disponível em: <http://www.scielo. br/pdf/rsp/v43n6/08.pdf>. Acesso em: 9 dez. 2011.

SILVA, R. J. S. et al. Prevalência e fatores associados à percepção negativa da saúde em pessoas idosas no Brasil. Revista Brasileira de Epidemiologia, São Paulo, v. 15, n. 1, p. 49-62, mar. 2012. Disponível em: <http//www.scielo.br/pdf/rbepid/v15n1/05.pdf>. Acesso em: 2 jun. 2012.

TRAD, L. A. B. et al. Estudo etnográfico da satisfação do usuário do Programa de Saúde da Família (PSF) na Bahia. Ciênciae SaúdeColetiva, Rio de Janeiro, v. 7, n. 3, p. 581-589, jul./set. 2002. Disponível em: $<$ http://www.scielo.br/scielo.php?script=sci_issuetoc\&pid=141 3812320020003\&lng=pt\&nrm=iso>. Acesso em: 12 mar. 2011.

VAN STRALEN, C. J. et al. Percepção dos usuários e profissionais de saúde sobre atenção básica: comparação entre unidades com e sem saúde da família na Região Centro-Oeste do Brasil. Cadernos de Saúde Pública, Rio de Janeiro, v. 24, supl. 1, p. 148-158, 2008. Disponível em: <http://www.scielo.br/pdf/csp/v24s1/19.pdf>. Acesso em: 10 jan. 2011.

VILLELA, W. V. et al. Desafios da atenção básica em saúde: a experiência de Vila Mariana, São Paulo, Brasil. Cadernos de Saúde Pública, Rio de Janeiro, v. 25, n. 6, p. 1316-1324, jun. 2009. Disponível em: <http://www.scielo.br/pdf/csp/v25n6/14.pdf>. Acesso em: 15 nov. 2011.

Recebido para publicação em Dezembro/2011

Versão final em Março/2013

Conflito de interesse: não houve

Suporte financeiro: inexistente 